\title{
QUERCETIN AMELIORATE INSULIN RESISTANCE CONCOMITANT EARLY CARDIOVASCULAR CHANGES IN EXPERIMENTAL RATS
}

\author{
AJAY SINGH KUSHWAH ${ }^{1 *}$ and GHANSHYAM DAS GUPTA ${ }^{2}$
}

'PhD Research Scholar, IK Gujral Punjab Technical University, Jalandhar, Kapurthala -144603, Punjab, India; and Department of Pharmacology, ASBASJSM College of Pharmacy, Bela-140111, Ropar, Punjab, India ${ }^{2}$ ISF Colleges of Pharmacy, Ghal Kalan, Ferozepur GT Road, Moga-142001, Punjab, India

\begin{abstract}
Quercetin is a dietary flavonoid found in a wide range of fruits and vegetables; it has diverse biological activities, possesses beneficial effects in ameliorating diabetic complications, apart from the effect of quercetin on fructose feed induced insulin resistance (IR) linked cardiac dysfunction have not been entirely revealed. This study aspires to explore the effect of quercetin on metabolism, oxidative stress, cardiomyocytes damage and cardiac function in IR state. Wistar rats either sex weighing 220-250 g ( $\mathrm{n}=8)$, were divided into four groups, kept on either control diet and high fructose diet and supplement with a quercetin as a test drug and metformin as a standard, at the dose of 50 and $200 \mathrm{mg} / \mathrm{kg}$; p.o., respectively. Daily measured body weight, feed, and water intake for 35 days, Oral glucose tolerance test (OGTT) performed in animals on the $32^{\text {nd }}$ day. At end of the study $\left(36^{\text {th }}\right.$ day), measured hemodynamic parameters after that estimation of various biochemical parameters. Finally, the animals were sacrificed for isolation of tissues and measured heart weight, the oxidative stress level of heart and histopathological changes. Treatment of quercetin with fructose-fed ameliorated all the parameters revile by the contrast of IR rats. The outcome of quercetin associated improves insulin sensitivity, normalized lipid profile, abolish hemodynamic changes, oxidative stress and cardiac injury markers within fructose-fed, and lesser histopathological changes were observed in contrast with IR rats. These beneficial effects of quercetin mediated by improving insulin sensitivity and metabolism; reduced oxidative stress could potentially be used to ameliorate the myocardial damage.
\end{abstract}

Keywords: quercetin, myocardium, oxidative stress, high-fructose diet, insulin resistance, 5' adenosine monophosphate-activated protein kinase

Various diseases emerge after the long exposure to Western diets as well as unhealthy lifestyle involving a lack of physical activity (1). Altering of diets has resulted in the large quantity of increase in daily intake of carbohydrates; as a major content of fructose having the main ingredient of these consumed foods (2). It has been recently confirmed that the consumption of high amounts of fructose in a form of food and beverage increases the risk lifestyle diseases such as hypertension, dyslipidemia, diabetes and obesity that act independently and synergistically (3). The relationship between high fructose intake and the development of insulin resistance (IR), exposure of the liver to a large amount of fructose leads to rapid stimulation of lipogenesis and triglyceride accumulation, which in turn contributes to reduced insulin sensitivity and hepat- ic IR or glucose intolerance (4). An assortment of fructose-fed rodent models reported allied with high triglyceride levels, adiposity, IR, and glucose intolerance associated with cardiovascular changes (5). Rather than cardiac IR include enhances pressure or volume overload, metabolic disparity, energy imperfection or decreased perfusion (6). This subsequently increases energy demand, reducing cardiac efficiency and metabolic derangements and damages the cardiomyocytes (7).

Therefore, several new drug treatment strategies have been developed in order to optimize these associative disorders; subsequently, maintain energy demand modification of patient complaints and perspectives of cardiovascular drug treatment with associated with diabetes in the population. AMPactivated protein kinase (AMPK) is a potential tar-

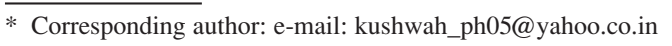


get for novel agents that may meet this challenge. AMP Kinase as an energy sensor that plays a key role in regulating cellular energy metabolism (8). In response to reduction of intracellular ATP levels (9), AMPK activates energy-producing pathways and inhibits energy-consuming processes: inhibits the protein, carbohydrate, and lipid biosynthesis, as well as cell growth and proliferation (10).

Salicylate a plant product has been used since ancient times as a medicinal use of molecule AMPK activator (11). Natural products such as Berberine, Quercetin, Phytosterol, Resveratrol, Epigallocatechin, and Capsaicin also exert glucose-lowering effects partly by activating AMPK through the regulation of mitochondrial function (10-12). Our investigational drug quercetin, it is most abundant dietary flavonoid found in a wide range of fruits and vegetables. Numerous studies reported that quercetin ameliorates oxidative stress (13), anti-inflammatory (14), prevent atherosclerosis (15) and retains endothelial function (16). Recently documented quercetin $(50 \mu \mathrm{M})$ treatment to L6 skeletal muscle cells, human $\mathrm{HepG}_{2}$ and murine $\mathrm{H}_{4}$ IIE hepatocytes for $18 \mathrm{~h}$ stimulated AMPK, increased $\mathrm{GLUT}_{4}$ translocation in cultured rat $\mathrm{L}_{6}$ skeletal muscle cells. It was also observed that quercetin induces hepatic AMPK activation and inhibits glucose-6-phosphatase in $\mathrm{H}_{4} \mathrm{IIE}$ hepatocytes. Moreover, quercetin exhibits capacity, though mildly, to enhance glycogen synthase in $\mathrm{HepG}_{2}$ hepatocytes which is a ratelimiting enzyme for glycogen synthesis (17). Selection of quercetin is based on the literature survey; it's involvement in activation of AMPK molecular switches that regulate energy balance of cell (18). The motive of this study was to examine the pharmacological evaluation of entity use of quercetin, as an AMPK activators as a novel therapeutic approach for treating of cardiovascular diseases associated with insulin resistance syndrome. Therefore, a new drug treatment scheme has been developed in direct help to prevent and to decrease the degree of myocardial injury in diabetes as well as to improve myocardial function. In view of these, quercetin could may be potentially be used as a drug to ameliorate cardiomyopathy induced by diabetes.

\section{MATERIAL AND METHODS}

\section{Drugs and chemicals}

Quercetin was procured from $\mathrm{Hi}$-media Mumbai and Metformin was obtained as gift sample from Swaroop Drug and Pharmaceuticals Maharashtra, India. Glucose, CK-MB and LDH kits were purchased from Recon diagnostic India. Alanine transaminase (ALT), Aspartate aminotransferase (AST), Alkaline phosphatase (ALP), Total cholesterol, Triglyceride level determined by commercially available kit by Span diagnostic, and uric acid determine by analysis kit by Accurex Biomedical Ltd.; as well as Serum insulin levels were determined by radioimmunoassay kit obtained from BRIT, BARC, Mumbai, India, all the other chemicals used were of analytical grade.

\section{Experimental animals and diets}

The study was conducted on 32 healthy albinos Wistar rats of either sex body weight 220-250 g were procured from registered breeder Disease free small animal house college of veterinary science, Hisar, Haryana, used in the study. They were housed well establish our institute central animal house facility appropriate polypropylene cages, lined with husk, renewed every $24 \mathrm{~h}$ under an environmentally controlled room temperature $\left(25 \pm 1^{\circ} \mathrm{C}\right)$ and relative humidity of $45-55 \%$ under $12: 12 \mathrm{~h}$ light/dark cycle and had free access to water and rodent food. All experiments were performed at the Department of Pharmacology ASBASJS Memorial College of Pharmacy, Bela, from 2014 to 2016. The whole experiment was carried out according to the guidelines of the Committee for the Purpose of Control and Supervision of Experiments on Animals (CPCSEA) New Delhi, India and approved by the Animal Ethics Committee (IAEC) this study was revived (Protocol No: ASCB/IAEC/07/14/91).

After 1 week of acclimatization period, the animal was planned for study, control group rats $(n=8)$ was fed a normal chow diet and rest of 24 rats were fed a fructose-enriched diet (Teklad-Harlan, Madison, USA formula) content provided $60 \%$ of total calories in the diet prepared in the laboratory with the following composition $(\mathrm{g} / \mathrm{kg}$ ) Casein high protein-207.0; DL-methionine-3.0; fructose-600.0; lard-50.0; cellulose-79.81; mineral mix-50.0; zinc carbonate-0.04; and vitamin mix-10, animals were maintained on these regimens for 5 weeks.

\section{Experimental design and study protocol}

Prior to dietary manipulation; all rats during acclimatization periods were fed standard pellet rodent diet. The rats were divided into four different groups $(\mathrm{n}=8)$ as under protocol design. Study group (C) normal chow feed control; group (F) fructosefed IR control, both groups rats were administered $0.9 \%$ normal saline $2 \mathrm{~mL}$ p.o; test groups $\left(\mathrm{F}+\mathrm{Q}_{50}\right)$, and standard group $\left(\mathrm{F}+\mathrm{M}_{200}\right)$ with fructose-fed rats received Quercetin 50 and Metformin $200 \mathrm{mg} / \mathrm{kg}$; p.o. respectively. Study group (C), received normal 
chow feed palates and rest of all study groups received fructose feed and treatment as per mentioned above. The doses were selected; test compound quercetin $(50 \mathrm{mg} / \mathrm{kg}$; p.o.), $(14,19)$ and as a standard metformin (dose $200 \mathrm{mg} / \mathrm{kg}$; p.o.) on the basis of previous literature review (20). Daily body weight feed and water intakes were measured and oral glucose tolerance test (OGTT) performed in animals on the $32^{\text {nd }}$ day. After performed OGTT, animals were supplemented with Lactated Ringer's Solution to prevent hypovolemia. Animals also supplemented with food and water ad libitum and treated with test dug as per schedule, discussed in the protocol. Animals were overnight fasted since $35^{\text {th }}$ day (to next $16 \mathrm{~h}$ ) on $36^{\text {th }}$ days animals were utilized to measure hemodynamic parameters. End of the experiments animals was sacrificed and blood was collected from the carotid artery for the assessment of the various biochemical measurements. Heart and liver tissue were collected to estimate isolate organ weight and to estimate heart tissue biomarkers and histopathological studies were carried out.

\section{Biochemical measurements}

The rats have fasted for $16 \mathrm{~h}$ and blood samples were collected and serums were prepared by centrifugation of the blood samples at $2000 \times \mathrm{g}$ for $20 \mathrm{~min}$ at $4^{\circ} \mathrm{C}$. Serum glucose levels, lipid profile (TGs, T-Chol, and HDL), liver function test (ALT, AST, and ALP) and uric acid, cardiac injury markers (CK-MB and LDH) were quantified using commercially available kits. Meanwhile, serum insulin levels were determined by standard radioimmunoassay technique using a standard kit obtained from BRIT, BARC, Mumbai, India experiment perform in Radio Immune Assay (RIA) laboratory. Following blood collection, rats were sacrificed and hearts were collected, weighed and stored for biochemical and histological analyses.

\section{Oral glucose tolerance test and insulin sensitivity index}

Oral glucose tolerance test (OGTT) was performed by measuring the glucose level in each group. The rats fasted for $16 \mathrm{~h}$ and blood samples were collected by retro-orbital plexus. A dose of 2 $\mathrm{g} / \mathrm{kg}$ (body weight) glucose solution (21) was given by gastric gavages. Blood samples were obtained from the retro-orbital plexus at pre 0 (before glucose administration), 30, 60, 90 and $120 \mathrm{~min}$. post glucose intake. Plasma glucose levels were measured by available commercial kits using auto bioanalyzer (Microlab 300).

From the blood, glucose and insulin levels, the degree of insulin resistance estimated by; using
Homeostasis Model Assessment (HOMA) as an index of insulin resistance, as calculated by the following formula: [Fasting serum insulin $(\mathrm{mU}) \times$ Fasting serum glucose ( mmol/L)/22.5] (22).

\section{Hemodynamic measurements}

At the end of the experimental period, rats were anesthetized with $25 \%$ urethane $(1.5 \mathrm{~g} / \mathrm{kg}$; i.p.). Throughout the experimental protocol body temperature of the animals was maintained at $37^{\circ} \mathrm{C}$. The neck was opened with a ventral midline incision to perform the tracheotomy. A polyethylene catheter $\left(\mathrm{PE}_{50}\right)$ filled with heparinized saline $(50 \mathrm{IU} / \mathrm{mL})$ and connected to a pressure transducer was inserted into the carotid artery. The signal was amplified by means of a bio-amplifier and monitored using the Power Lab system (AD Instruments, NSW Australia). The measurement of systolic blood pressure (SBP), diastolic blood pressure (DBP), arterial blood pressure (ABP), mean arterial blood pressure (MAP) and heart rate (HR) were then obtained.

Determination of lipid peroxidation (LPO), superoxide dismutase (SOD), catalase (CAT) and glutathione peroxidase (GPx) levels in heart homogenates

After blood collection for hemodynamic measurements, the animal was sacrificed hart tissue harvest; blood was washed off from the heart chambers by using ice-chilled physiological saline. The heart was then thawed, placed in $10 \%$ tissue homogenate buffer which was prepared using a phosphate buffer (0.1 M, pH 7.4). The homogenates were centrifuged at $600 \times \mathrm{g}$ for $10 \mathrm{~min}$ to remove cell debris. The supernatant was again centrifuged at $10,000 \times \mathrm{g}$ for $30 \mathrm{~min}$ to obtain the cytosolic fraction. All procedures were carried out at $4^{\circ} \mathrm{C}$. The supernatants were then used for biochemical analyses.

Malondialdehyde (MDA), a measurement of LPO in the heart was assayed in the form of thiobarbituric acid-reactive substances (TBARS) following the method of Ohkawa et al. (23). The rate of lipid peroxidation was expressed as mmol of MDA formed/g wet weight tissue. SOD activity level was determined according to the method of Mishra and Fridovich (24) and was expressed as the amount of enzyme that inhibits epinephrine oxidation by $50 \%$, equal to $1 \mathrm{U} / \mathrm{mg}$ of protein. CAT activity level was determined from the decomposition of $\mathrm{H}_{2} \mathrm{O}_{2}$ at 240 $\mathrm{nm}$ for 3 min and was monitored by using a spectrophotometer, followed the method of according to Maehly and Chance (25). The activity level of this enzyme was expressed in mmol $\mathrm{H}_{2} \mathrm{O}_{2}$ metabolized/mg protein /min. GPx activity level was deter- 
mined according to the method of Rotruck et al., (26) and was expressed as mM of GSH consumed /mg protein/ min.

\section{Histology}

The animals were sacrificed; the heart was isolated, washed with ice-cold saline and fixed in $10 \%$ buffered formalin for $24 \mathrm{~h}$. The samples were sent pathology department of IVY hospital, sec-71, S.A.S Nagar (Mohali) for the preparation of slides of heart tissue. Tissues were dehydrated in a graded series of alcohol, embedded in paraffin, sectioned in $5 \mathrm{~mm}$ thickness by using a microtome. The sections were dewaxed in three changes of xylene, hydrated in two changes of $100 \%$ ethanol, followed by $95 \%$ and $80 \%$ ethanol, rinsed with water and then stained with hematoxylin and eosin ( $\mathrm{H}$ and $\mathrm{E})$. For interpretation, the pictures were taken from the prepared slides with the help of photomicroscope.

\section{Statistical analysis}

All datasets were represented as the mean \pm standard error of the mean (SEM). Comparisons of findings between groups were made via statistical analysis of data sets using one-way analysis of variance (ANOVA). When interaction and/or the main effects were significant, means were compared using the Newman-Keuls multiple-comparison post$h o c$ test. A $p$-value of $<0.05$ was considered statistically significant. All statistical analyses were performed using Graph Pad Prism version 5.00 for Windows.

\section{RESULTS}

Effects of Quercetin on body weight; feed and water intake and organ somatic weight

At the beginning of the study, there was no significant difference in mean body weight, food intake and water intake among all groups. Fructose fed-IR rats significantly reducing the weight gain $(\mathrm{p}<$ 0.001) in the final body weight when compared to the initial body weight and also significantly decreasing the feed intake $(\mathrm{p}<0.01)$ when compared to normal chow diet rats. Meanwhile, in IR control group rats do not show remarkable changes in water intake

Table 1. Effect of Quercetin on \% weight gain, food and water intake, and organs somatic index.

\begin{tabular}{|l|c|c|c|c|}
\hline \multicolumn{1}{|c|}{ Parameters } & $\mathrm{C}$ & $\mathrm{F}$ & $\mathrm{F}+\mathrm{Q}_{50}$ & $\mathrm{~F}+\mathrm{M}_{200}$ \\
\hline \multicolumn{5}{|c|}{ Body weight, food and water intake } \\
\hline \% Weight gain at week 5(g) & $13.13 \pm 7.07$ & $2.84 \pm 4.43^{* * *}$ & $4.56 \pm 2.41$ & $4.58 \pm 3.63$ \\
\hline Food intake at week 5(g/day) & $20.29 \pm 1.06$ & $9.09 \pm 0.85^{* *}$ & $11.11 \pm 0.79$ & $10.17 \pm 0.81$ \\
\hline Water intake at week 5 (mL/day) & $25.45 \pm 0.89$ & $27.57 \pm 1.40$ & $26.71 \pm 0.58$ & $25.71 \pm 0.43$ \\
\hline \multicolumn{7}{|c|}{ Organs somatic index (W/W \%) } \\
\hline Liver weight in (g) & $4.08 \pm 0.18$ & $5.84 \pm 0.17^{*}$ & $4.21 \pm 0.18^{\#}$ & $3.89 \pm 0.13^{\#}$ \\
\hline Heart weight in (g) & $0.27 \pm 0.63$ & $0.31 \pm 0.67^{*}$ & $0.28 \pm 0.08^{\#}$ & $0.26 \pm 0.01^{\# \#}$ \\
\hline
\end{tabular}

Study group (C) normal chow feed control; group (F) fructose-fed IR control, both groups rats were administered $0.9 \%$ normal saline 2 $\mathrm{mL}$ p.o; test groups $\left(\mathrm{F}+\mathrm{Q}_{50}\right)$, and standard group $\left(\mathrm{F}+\mathrm{M}_{200}\right)$ with fructose-fed rats received Quercetin 50 and Metformin $200 \mathrm{mg} / \mathrm{kg}$; p.o respectively. (Values expressed as mean $\pm \mathrm{SEM}, \mathrm{n}=8$ ). ${ }^{*} \mathrm{p}<0.05$, ${ }^{* *} \mathrm{p}<0.01$, *** $\mathrm{p}<0.001$ when compared with $\mathrm{C}$; ${ }^{*} \mathrm{p}<0.05$, ${ }^{* *} \mathrm{p}<0.01$ when compared with F; One way ANOVA followed by Newman-Keuls comparison test

Table 2. Effects of Quercetin on fasting serum glucose, insulin level; HOMA of insulin resistance index, OGTT, and AUC.

\begin{tabular}{|l|c|c|c|c|}
\hline \multicolumn{1}{|c|}{ Parameters } & $\mathrm{C}$ & $\mathrm{F}$ & $\mathrm{F}+\mathrm{Q}_{50}$ & $\mathrm{~F}+\mathrm{M}_{200}$ \\
\hline Glucose $(\mathrm{mg} / \mathrm{dL})$ & $94.11 \pm 1.28$ & $130.1 \pm 3.56^{* * *}$ & $117.1 \pm 2.64^{\#}$ & $103.1 \pm 1.44^{\# \#}$ \\
\hline Insulin $(\mu \mathrm{U} / \mathrm{mL})$ & $27.29 \pm 2.39$ & $48.36 \pm 5.20^{* * *}$ & $30.57 \pm 3.80^{*}$ & $28.73 \pm 2.60^{\#}$ \\
\hline HOMA $(\%$ of control group) & $6.34 \pm 1.53$ & $15.53 \pm 2.67 * * *$ & $8.83 \pm 1.15^{\#}$ & $7.31 \pm 2.32^{\# \#}$ \\
\hline OGTT AUC $(\mathrm{mmol} / \mathrm{L})$ & $18084 \pm 4.31$ & $21309 \pm 7.93^{* *}$ & $16088 \pm 1.34^{\# \#}$ & $15721 \pm 1.06^{\# \#}$ \\
\hline
\end{tabular}

Abbreviations: (HOMA) Homeostasis model assessment of insulin resistance index; (OGTT AUC) Oral glucose tolerance test Area under the curve. Study group (C) normal chow feed control; group (F) fructose-fed IR control, both groups rats were administered $0.9 \%$ normal saline $2 \mathrm{~mL}$ p.o; test groups $\left(\mathrm{F}+\mathrm{Q}_{50}\right)$, and standard group $\left(\mathrm{F}+\mathrm{M}_{200}\right)$ with fructose-fed rats received Quercetin 50 and Metformin 200 $\mathrm{mg} / \mathrm{kg}$; p.o respectively. (Values expressed as mean $\pm \mathrm{SEM}, \mathrm{n}=8$ ). ${ }^{* *} \mathrm{p}<0.01$, ${ }^{* * *} \mathrm{p}<0.001$ when compared with $\mathrm{C}$; ${ }^{*} \mathrm{p}<0.05$, "\# $<0.01$ when compared with F; One way ANOVA followed by Newman-Keuls comparison test 
Table 3. Effects of Quercetin on lipid profile, liver functions tests and uric acid level.

\begin{tabular}{|c|c|c|c|c|}
\hline Parameters & $\mathrm{C}$ & $\mathrm{F}$ & $\mathrm{F}+\mathrm{Q}_{50}$ & $\mathrm{~F}+\mathrm{M}_{200}$ \\
\hline \multicolumn{5}{|c|}{ Lipid profile } \\
\hline TGs (mg/dL) & $62.29 \pm 5.02$ & $153.8 \pm 12.08 * * *$ & $113.9 \pm 9.21^{\# \#}$ & $87.0 \pm 4.765^{\# \# \#}$ \\
\hline T-Chol (mg/dL) & $55.21 \pm 3.80$ & $119.65 \pm 4.54 * * *$ & $99.92 \pm 4.46^{\#}$ & $72.29 \pm 5.02^{\# \#}$ \\
\hline HDL-C (mg/dL) & $41.33 \pm 1.51$ & $11.31 \pm 0.64 * * *$ & $25.31 \pm 1.42^{\#}$ & $33.62 \pm 3.12^{\# \#}$ \\
\hline \multicolumn{5}{|c|}{ Liver functions test } \\
\hline ALT (IU/L) & $27.46 \pm 1.13$ & $78.09 \pm 1.75^{* * *}$ & $44.77 \pm 1.23^{\# \#}$ & $38.32 \pm 0.47^{\# \# \#}$ \\
\hline AST(IU/L) & $18.23 \pm 1.44$ & $41.23 \pm 0.85^{* *}$ & $36.77 \pm 0.92^{\#}$ & $23.98 \pm 1.49^{\# \#}$ \\
\hline ALP(IU/L) & $134.72 \pm 2.2$ & $221.1 \pm 6.43^{* * *}$ & $188.43 \pm 3.66^{\#}$ & $142.9 \pm 1.79^{\# \#}$ \\
\hline Uric Acid(mg/dL) & $1.96 \pm 0.21$ & $5.47 \pm 0.25^{* * *}$ & $3.40 \pm 0.04^{\#}$ & $4.90 \pm 0.26$ \\
\hline
\end{tabular}

Abbreviations: (TGs) Triglycerides, (T-Chol) Total cholesterol, (HDL-C)High-density lipoprotein cholesterol, (ALT) Alanine transaminase, (AST) Aspartate transaminase, (ALP) Alkaline Phosphatase. Study group (C) normal chow feed control; group (F) fructose-fed IR control, both groups rats were administered $0.9 \%$ normal saline $2 \mathrm{~mL}$ p.o; test groups $\left(\mathrm{F}+\mathrm{Q}_{50}\right)$, and standard group $\left(\mathrm{F}+\mathrm{M}_{200}\right)$ with fructose-fed rats received Quercetin 50 and Metformin $200 \mathrm{mg} / \mathrm{kg}$; p.o respectively. (Values expressed as mean \pm SEM, $\mathrm{n}=8$ ). $* *$ p $<0.01$, ${ }^{* * *} \mathrm{p}<0.001$ when compared with $\mathrm{C}$; ${ }^{\mathrm{p}} \mathrm{p}<0.05,{ }^{*} \mathrm{p}<0.01,{ }^{* * * *} \mathrm{p}<0.001$ when compared with F; One way ANOVA followed by NewmanKeuls comparison test

when compared to normal chow diet rats. As similar in fructose-fed treatment groups with quercetin and metformin, body weight gain, feed, and water intake do not show any remarkable changes when compared with the fructose feeding IR rats (Table 1).

Liver and cardiac somatic index in normal, in chow diet rats and quercetin treatment, did not cause significant changes in the somatic index. In fructose-fed IR rats, heart and liver somatic index markedly increase $(\mathrm{p}<0.05)$ when compared to normal chow diet rats. In fructose-fed rats treatment with quercetin and metformin resulted significantly decrease in heart and liver somatic index $(p<0.05)$ when compared to fructose-fed IR rats (Table 1).

Effects of Quercetin on fasting serum glucose, insulin level; Insulin resistance index and oral glucose tolerance test (OGTT)

Fructose feeding for five weeks in rats showed significantly $(\mathrm{p}<0.001)$ increased the level of fasting serum glucose and insulin as compared to chow-fed rats. Treatment with quercetin and metformin in fructose-fed rats significantly $(\mathrm{p}<0.05)$ and $(\mathrm{p}<0.01)$ prevented the increased serum glucose with respect of treatment and insulin level in both treatments with fructose-fed rats significantly $(\mathrm{p}<0.05)$ prevented the raised serum insulin with respect of treatment as compared with fructose-fed IR rats (Table 2).

Fructose feeding in IR group showed increased ( $\mathrm{p}<0.001)$ HOMA-IR levels as compared to chowfed rats. Treatment with quercetin and metformin in fructose-fed rats significantly $(\mathrm{p}<0.05)$ and $(\mathrm{p}<$ 0.01 ) prevented the elevation HOMA-IR levels with respect of treatment as compared to fructose-fed IR rats (Table 2). Study of the OGTT (Fig. 1) and the comparison between total areas under the curve of glycemia evidenced that fructose-fed IR rats developed glucose intolerance assessed by significantly $(\mathrm{p}<0.01)$ increased the total area under curve values after glucose administration for as compared to chow-fed rats. Fructose-fed rat treatment with quercetin and metformin significantly $(p<0.01)$ prevented the elevation in total area under curve values due to glucose administration when compared to fructose-fed IR control rats (Table 2).

Effects of Quercetin on lipid profile, liver functions test and uric acid level

Fructose feeding in IR group, lipid profile changes significantly increased $(p<0.001)$ TGs and $\mathrm{T}-\mathrm{Chol}$, and significantly decrease $(\mathrm{p}<0.001)$ serum HDL-C level as compared to chow-fed rats. Treatment with quercetin and metformin in fructosefed IR rats significantly $(p<0.01)$ and $(p<0.001)$ decrease serum TGs, with respect of treatment and also prevent the elevation of T-chol significantly ( $\mathrm{p}$ $<0.05)$ and $(\mathrm{p}<0.01)$ with respect to treatment, rather than similar treatment group elevate significantly $(\mathrm{p}<0.05)$ and $(\mathrm{p}<0.01)$ serum HDL-C when compared to IR control rats (Table 3 ).

Fructose enriches diet IR rats affect the liver function enzymes ALT, ALP and also affected the serum uric acid levels, increase significantly $(\mathrm{p}<$ $0.001)$ and in the similar group, AST level rise significantly $(p<0.01)$ as compared to chow-fed rats. Effects of treatment with quercetin and metformin 
on fructose-fed rats, ALT level reduced significantly $(p<0.01)$, $(p<0.001)$ and the level of AST significantly decreased $(\mathrm{p}<0.05),(\mathrm{p}<0.01)$ and also prevent the elevation of level of ALP $(p<0.05)$, $(\mathrm{p}<0.001)$ with respect of treatment when compare with fructose-fed IR control rats. Findings of study in treatment with quercetin in fructose enrich diet on uric acid levels, significantly reduced $(\mathrm{p}<0.05)$, but metformin treatment in similar manner diet did not significantly reduce the uric acid level when compared with fructose-fed IR control rats (Table 3).

\section{Effects of Quercetin on levels of serum cardiac injury markers}

Serum levels of CK-MB and LDH in fructosefed IR rats were higher $(\mathrm{p}<0.01)$ observed when compared to normal chow diet rats. Treatment with quercetin and metformin on fructose-fed rats resulted in markedly reduced $(\mathrm{p}<0.05),(\mathrm{p}<0.01)$ and serum LDH levels remarkable $(\mathrm{p}<0.05)$ prevented elevation with respect of treatment when compared to fructose-fed IR control group rats (Table 4).

Effects of Quercetin on hemodynamic parameters

Fructose enrich diet affect the SBP were significantly $(\mathrm{p}<0.001)$ increased and also DBP, MAP, and HR remarkably $(\mathrm{p}<0.01)$ raised in fructose-fed
IR rats, but ABP slightly increases $(\mathrm{p}<0.05)$ when compared with normal chow diet rats. Meanwhile, treatment of fructose-fed rats with quercetin and metformin alter the SBP; significantly decrease $(p<0.01)$ with respect to treatment; however DBP, ABP, MAP, and HR with both treatments significantly reduce $(p<0.05)$ with respect to treatment, when compared with fructose-fed IR rats (Table 4).

\section{Effects of Quercetin on oxidative stress level in the heart}

Fructose diet affects the Oxidative stress level in the heart in contest of it LPO was significantly elevated $(\mathrm{p}<0.001)$ and decrease in the SOD, CAT level significantly $(\mathrm{p}<0.05)$ and also GPx level remarkably $(\mathrm{p}<0.001)$ decrease in fructose-fed IR control group when compared with normal chow diet rats. In the fructose-fed rat, treated with quercetin reduce the LPO level significantly $(p<0.01)$ and rise near to normal level SOD, CAT level significantly $(\mathrm{p}<0.05)$ and also GPx level remarkably $(\mathrm{p}<0.01)$ increase when compared with fructosefed IR rats. Standard treatment with metformin reduces the LPO and rise the near to normal level GPx, CAT significantly ( $p<0.05$ ) but no remarkable changes in SOD level observed when compared with fructose-fed IR rats (Table 4).

Table 4. Effects of Quercetin on serum cardiac injury markers, hemodynamic parameters and oxidative stress level in heart.

\begin{tabular}{|c|c|c|c|c|}
\hline Parameters & $\mathrm{C}$ & $\mathrm{F}$ & $\mathrm{F}+\mathrm{Q}_{50}$ & $\mathrm{~F}+\mathrm{M}_{200}$ \\
\hline \multicolumn{5}{|c|}{ Serum cardiac injury markers } \\
\hline CK-MB(IU/L) & $63.05 \pm 1.06$ & $92.08 \pm 0.92 * *$ & $75.78 \pm 1.08^{\#}$ & $66.28 \pm 1.45 \# \#$ \\
\hline $\mathrm{LDH}(\mathrm{IU} / \mathrm{L})$ & $790.2 \pm 9.2$ & $963.3 \pm 8.0 * *$ & $903.3 \pm 8.4^{\#}$ & $803 \pm 14.84^{\#}$ \\
\hline \multicolumn{5}{|c|}{ Hemodynamic parameters } \\
\hline $\mathrm{SBP}(\mathrm{mmHg})$ & $113.8 \pm 1.10$ & $146.6 \pm 3.04 * * *$ & $118.5 \pm 2.62^{\#}$ & $117.2 \pm 3.13^{\# \#}$ \\
\hline $\mathrm{DBP}(\mathrm{mmHg})$ & $85.24 \pm 0.97$ & $103.6 \pm 1.241 * *$ & $95.94 \pm 1.71^{\#}$ & $92.71 \pm 2.33^{\#}$ \\
\hline $\mathrm{ABP}(\mathrm{mmHg})$ & $102.52 \pm 0.77$ & $139.40 \pm 0.611^{*}$ & $110.74 \pm 1.52^{\#}$ & $106.53 \pm 1.38^{\#}$ \\
\hline MAP (mmHg) & $94.76 \pm 0.69$ & $117.93 \pm 0.827^{* *}$ & $102.45 \pm 1.149^{*}$ & $106.0 \pm 0.98^{\#}$ \\
\hline $\mathrm{HR}(\mathrm{BPM})$ & $377.05 \pm 1.70$ & $419.41 \pm 1.93^{* *}$ & $396.31 \pm 0.56^{\#}$ & $389.33 \pm 3.76^{\#}$ \\
\hline \multicolumn{5}{|c|}{ Oxidative stress level in heart } \\
\hline LPO (nM MDA/mg of protein) & $1.38 \pm 0.12$ & $20.19 \pm 1.24 * * *$ & $9.38 \pm 0.52^{\# \#}$ & $13.56 \pm 0.64^{\#}$ \\
\hline SOD (U/mg of protein) & $7.34 \pm 0.02$ & $5.39 \pm 0.06^{*}$ & $6.23 \pm 0.09^{\#}$ & $5.86 \pm 0.07$ \\
\hline CAT (nM/mg of protein/min) & $4.201 \pm 0.02$ & $1.43 \pm 0.07 *$ & $2.53 \pm 0.07^{\#}$ & $2.35 \pm 0.05^{\#}$ \\
\hline GPx (nM/mg of protein/min) & $19.59 \pm 0.570$ & $5.11 \pm 0.48 * * *$ & $12.58 \pm 0.57^{\# \#}$ & $9.61 \pm 0.61^{\#}$ \\
\hline
\end{tabular}

Abbreviations: (CK-MB) Creatine kinase isoenzyme, (LDH) Lactate dehydrogenase, (SBP) Systolic blood pressure, (DBP) Diastolic blood pressure, (MAP) Mean arterial pressure, (ABP) Arterial blood pressure, (HR) Heart Rate, (LPO) Lipid peroxidation, (MDA) Malondialdehyde, (SOD) Superoxide dismutase, (CAT) Catalase, (GPx) Glutathione peroxidase levels. Study group (C) normal chow feed control; group (F) fructose-fed IR control, both groups rats were administered $0.9 \%$ normal saline $2 \mathrm{~mL}$ p.o; test groups $\left(\mathrm{F}+\mathrm{Q}_{50}\right)$, and standard group $\left(\mathrm{F}+\mathrm{M}_{200}\right)$ with fructose-fed rats received Quercetin 50 and Metformin $200 \mathrm{mg} / \mathrm{kg}$; p.o respectively. (Values expressed as mean \pm SEM, $\mathrm{n}=8$ ). ${ }^{*} \mathrm{p}<0.05,{ }^{* *} \mathrm{p}<0.01,{ }^{* * *} \mathrm{p}<0.001$ when compared with $\mathrm{C} ;{ }^{*} \mathrm{p}<0.05,{ }^{*} \mathrm{p}<0.01$ when compared with F; One way ANOVA followed by Newman-Keuls comparison test 


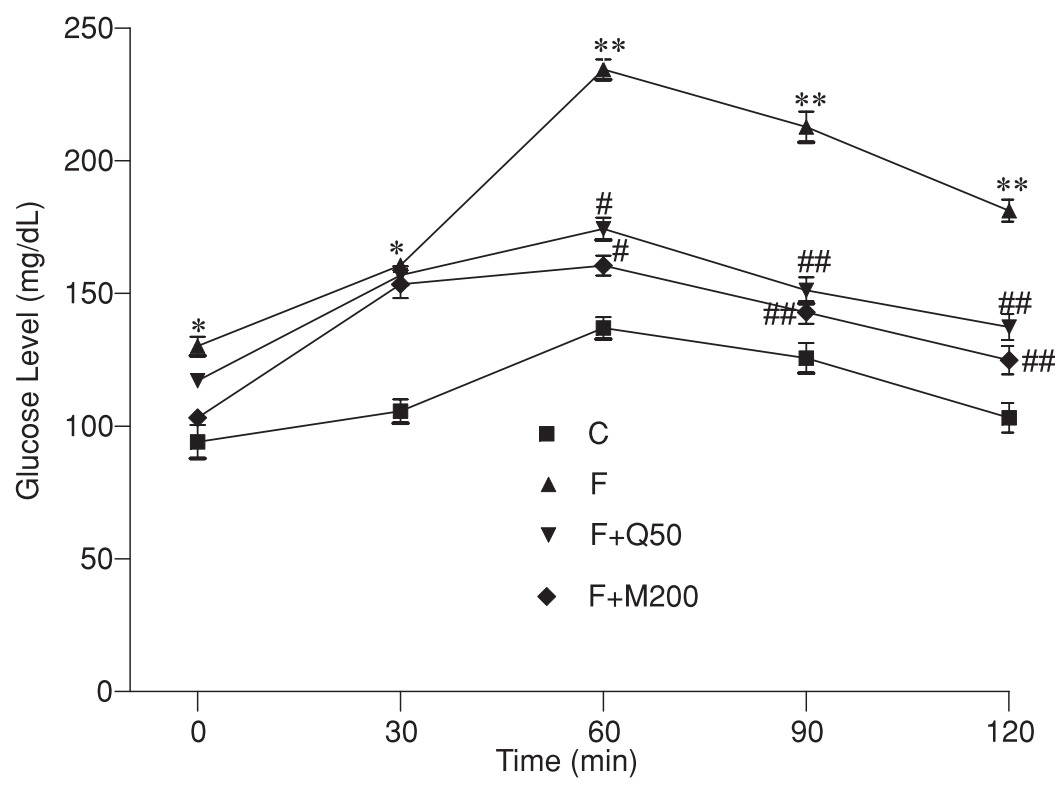

Figure 1. Effects of Quercetin on Oral glucose tolerance test (OGTT)

Study group (C) normal chow feed control; group (F) fructose-fed IR control, both groups rats were administered $0.9 \%$ normal saline 2 $\mathrm{mL}$ p.o; test groups $\left(\mathrm{F}+\mathrm{Q}_{50}\right)$, and standard group $\left(\mathrm{F}+\mathrm{M}_{200}\right)$ with fructose-fed rats received Quercetin 50 and Metformin $200 \mathrm{mg} / \mathrm{kg}$; p.o respectively. (Values expressed as mean \pm SEM., $\mathrm{n}=8$ ). ${ }^{*} \mathrm{p}<0.05$, ${ }^{* *} \mathrm{p}<0.01$ when compared with $\mathrm{C}$; ${ }^{*} \mathrm{p}<0.05$, ${ }^{\#} \mathrm{p}<0.01$ when compared with F; One way ANOVA followed by Newman-Keuls comparison test

\section{Effects of Quercetin on histopathological changes in the rat liver and heart}

Fructose-fed IR rats (Fig. 2b) induced histopathological changes in rat liver including edema; necrosis and hepatocytes degenerations were severe when compared to normal chow diet group (Fig. 2a). The changes in quercetin and metformin treatment (Figs. 2c-d) respectively in fructose-fed rats moderately decreased when compared with the fructosefed IR control group (Fig. 2b).

In normal chow diet rats (Fig. 3a) cardiac muscle fibers were found to be of uniform size, shape, and configurations with no inflammatory cell infiltrate. Fructose-fed IR (Fig. 3b) rats produced massive change in the myocardium showing a varying degree of vascular changes in the cardiac muscle, in present study treatment with quercetin and metformin (Figs. 3c-d) respectively, inhibits fructose feed induced cardiac damage by reversal of infiltration of inflammatory cells and fragmentation of myofibrils and contraction band necrosis.

\section{DISCUSSION}

Chronic provide to eat carbohydrates rich diet in a form of fructose, induce the hypertriglyceridemia, hyperinsulinemia, and rise in the blood pressure; these are cardinal features of insulin resist- ance (IR) (27). Results of the present study confirm these observations as the feeding of a fructose-rich diet for 35 days raised glucose, insulin, triglyceride levels; these are key features of syndrome "X". Development of hypertension is secondary to hyperinsulinemia in fructose-fed IR rats and it is evident in this study. The importance of this model can be gauged from the fact that it has been used for assessing the therapeutic efficacy of presently available insulin sensitizers; therefore this animal model was selected for the study. Several new drug treatment strategies have been developed in order to optimize these associative disorders; subsequently, maintain energy demand modification of patient complaints and perspectives of cardiovascular drug treatment associated with diabetes in the population. A number of study reference with some or all signs being reversed when natural compounds from foods, including flavonoids, such as Rutin and Naringin, are added to this diet (28). Flavones, especially quercetin is widely distributed in plants and is present in considerable amounts in fruits and vegetables. In addition to their anti-oxidant effect, it interferes with a large number of biochemical signaling pathways, physiological and pathological processes (13-16).

Result findings in high fructose-fed IR groups consumed less daily food intake and gained signifi- 
cantly less weight compared to the chow-fed control group. Fructose-rich diet IR rats make the reduction in body weight gain could be due to the ability of rats to adapt for the excess calories obtained when consuming $60 \%$ fructose by taking in fewer calories thereby maintaining a normal body weight (29), consistent in normal, rats gained weight when their chow diet was supplemented. The lesser gain of body weight in fructose-fed rats receiving quercetin with the comparison of fructose IR control rats should be due to near normal fasting blood glucose and serum insulin levels. Chronic consumption of fructose-enriched diet resulted in the development of insulin resistance, evident by impairment of elevations of fasting blood glucose and intolerance, an increase of HOMA-IR index. Higher insulin levels in fructose-fed IR rats' decreases catabolism of fats and glycogen, which subsequently prevented the decreased in organs' weight. This could explain higher cardiac and liver somatic index observed in fructose-fed IR rats. These studies prove that quercetin maintains normal cardiac and liver somatic index by improved insulin sensitivity when compared to fructose-fed IR rats. It is indicated by significant decreases in OGTTAUC, and HOMA-IR index was supported by recently study documented treatment of quercetin on skeletal muscle cells and hepatocytes stimulated AMPK and increased GLUT $_{4}$ translocation in cultured rat $\mathrm{L}_{6}$ skeletal muscle cells and improved insulin sensitivity (17).

High fructose intake and the development of IR, lead to exposure of the liver to a huge amount of fructose, accelerating lipogenesis and triglyceride accumulation, which in turn contributes to reduced hepatic insulin sensitivity, glucose intolerance, and dysfunction (4). The study confirms that fructoserich diet rise TGs and T-Chol level and decreasing of HDL-C these are cardinal features of etiology of hepatic and cardiac dysfunctions. Moreover, another study documented that isoquercetin and Quercetin-3-O-gentiobioside treated mice showed the reduction in cholesterol levels and expression of nuclear receptor transcription factor PPAR $\gamma$, which is an important regulator of lipid and glucose homeostasis (30). These studies prove that quercetin produces protective action in high carbohydrate-fed induced lipid dysfunction in rats. The constant hyperlipidemic state increased the lipid accumulation and enzymes dysfunction thus promotes the intrahepatocellular lipids deposition and the cause
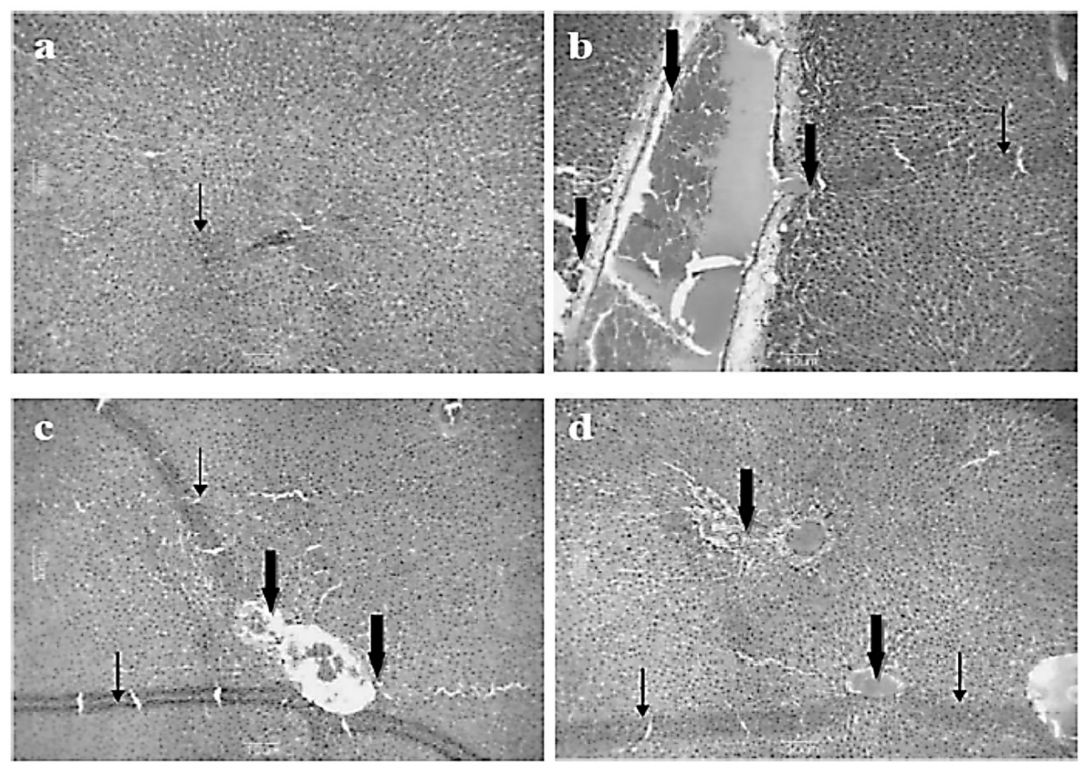

Figure 2 (a-d): Effects of Quercetin on Histopathological changes in the various study groups of rat liver tissues.

Representative images for histopathological examination of livers of rats in (a) control study group (C) normal chow feed control; (b) group (F) fructose-fed IR control, both groups rats were administered $0.9 \%$ normal saline $2 \mathrm{~mL}$ p.o; (c) test groups $\left(\mathrm{F}+\mathrm{Q}_{50}\right)$ and $(\mathrm{d})$ standard group $\left(F+M_{200}\right)$ with fructose-fed rats were receives Quercetin 50 and Metformin $200 \mathrm{mg} / \mathrm{kg}$; p.o respectively. Liver sections were fixed and stained with hematoxylin and eosin ( $\mathrm{H}$ and E) stain. Images were taken at $100 \times$ magnification and scale bar $=10 \mathrm{M}$. Figure (a) showed the normal histological appearance of the liver hepatocytes and central vein and hepatocytes were arranged in a network around the central vein (Thin arrow); in figure (b) (Bold arrows) showed, edema and necrosis of hepatocytes in the liver cytoplasm. Figure (c) showed less edema and necrotic changes. In figure (d) show, considerably mild hepatic changes when compared to (a) figures and lesions were localized in some liver lobules in form of degenerative cell foci with fewer cells 


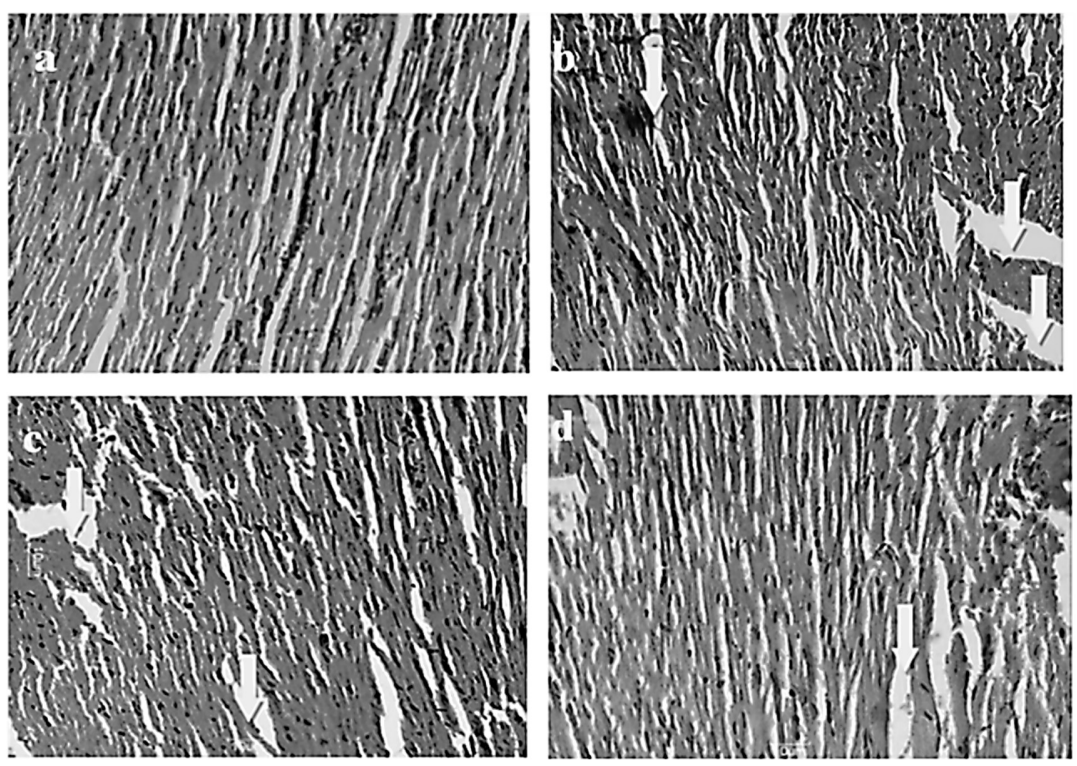

Figure 3 (a-d): Effects of Quercetin on Histopathological changes in the various study groups of rat heart tissues. Representative images for histopathological examination of the heart of rats in (a) control study group (C) normal chow feed control; (b) group (F) fructose-fed IR control, both groups rats were administered $0.9 \%$ normal saline $2 \mathrm{~mL}$ p.o; (c) test groups $\left(\mathrm{F}+\mathrm{Q}_{50}\right)$ and $(\mathrm{d})$ standard group $\left(\mathrm{F}+\mathrm{M}_{200}\right)$ with fructose-fed rats received Quercetin 50 and Metformin $200 \mathrm{mg} / \mathrm{kg}$; p.o respectively. Heart sections were fixed and stained with hematoxylin and eosin $(\mathrm{H}$ and $\mathrm{E})$ stain. Images were taken at $100 \times$ magnification and scale bar $=10 \mathrm{M}$. Figure (a) showed clear integrity of myocardial cell membrane; in figure (b) examination of the visibly marked myonecrosis, edema, muscle separation and infiltration of inflammatory cells were observed (arrow). The reduction of histopathological scores in figure (c) and also show less edema and necrotic (arrow) changes and figure (d) show, histological protection of the myocardium

may lead to endothelial dysfunction (31). Hepatic ALT, AST, and ALP enzymes are the marker of sensing of liver damage and these enzymes levels rise in serum are predictive of damage to the liver. This declaration already holds in the result of an assessment of fructose feeding IR rats liver histopathological slides. In our findings, high fructose diet IR group animals have significantly increased the level of the liver damage marker in the comparison of normal chow diet control group. The reduced activities of the serum biomarker enzymes and enzymatic leakage in the liver may be due to an enhanced antioxidant potential which could be responsible for attenuating lipid-peroxidation and reactive oxygen species induced oxidative stress and inflammation (32). Many investigations have confirmed the hyperuricemia occurs with metabolic syndrome. Uric acid may simply be a consequence of the increased uric acid absorption in the proximal tubule secondary to hyperinsulinemia there is growing data that uric acid may predict the development of metabolic syndrome, obesity, and diabetes (33). In these studies, IR animals treated with high fructose feed increase in uric acid as compared with normal chow diet group. It has been hypothesized that uric acid overproduction can trigger oxidative stress xanthine oxidoreductase (XO), the enzyme responsible for urate formation, may play a critical role in hyperuricemia and reduce the activity of nitric oxide synthase in metabolic syndrome (34). Quercetin treatment improved parameters associated with renal dysfunction possibly due to the presence of antioxidant properties (35).

Links in between IR and heart failure occurrence of a cardiomyopathy is now recognized. Cardiac insulin resistance is characterized by the reduced availability of sarcolemmal GLUT transporters and consequent lower glucose exhibit cardiac hypertrophy and marked excitation-contraction coupling abnormalities were characterized (5-7). A shift away from glycolysis towards fatty acid oxidation for ATP supply is apparent and is associated with myocardial oxidative stress (36). In response to reduction of intracellular ATP levels, AMPK activates energy-producing pathways and inhibits energy-consuming processes (9-10). Abnormalities in metabolism can cause an increase in intracellular reactive oxygen species (ROS) levels that leading to decrease anti-oxidative defenses mechanism it is the main causes of the cells injury (37). GSH has a direct antioxidant function by reacting with superoxide radicals and formation of oxidized GSH. It 
plays an important role in the regulation of a cell protection against oxidative injury. MDA is the stress marker that indicates lipid per-oxidation of endogenous lipid has been shown to be a major risk factor leading to myocardial injury in metabolic syndrome (38). When myocardial cells are damaged, leakage of myocardial enzymes such as LDH and $\mathrm{CK}-\mathrm{MB}$ occurs which accounts for the increased activities of serum biomarker enzymes and therefore, serve as a sensitive index to assess the severity of irreversible myocardial injury LDH converts pyruvate, the final product of glycolysis to lactate when oxygen is absent or in short supply and the activity of LDH will increase when ischemia lasts (39). In un-treated fructose-fed IR rats markedly affect hemodynamic parameters, could be due to loss of normal heart muscle architecture, abnormal cardiac physiology and the loss of glycogen (40).Consumption of fructose-enriched diet in rats able to increases oxidative stress level in heart, and also the initiation of tissue injury were noted necrosis, loss of normal heart muscle architecture and also reported cardiac injury markers in serum. In concordant with these findings, histopathological changes indicating of tissue injury were noted these were reflected by abnormal hemodynamic characters.

Quercetin treatment in fructose-fed rats was found able to restore the activity level of antioxidative enzymes including SOD, CAT, and GPx as well as ameliorating levels of lipid peroxidation product in fructose-fed rat's heart. Quercetin was able to decrease injury to the cardiac muscles in fructose-fed rats. The levels of CK-MB and $\mathrm{LDH}$ fructose-fed rats were markedly decreased following quercetin treatment additionally, has been reported to possess free radical scavenger activity and able to activate cellular antioxidative enzyme levels and will be stored the normal cell structure in the heart $(41,42)$. Preservation of near normal heart muscle architecture will help to preserve near normal heart functions these were reflected by near normal SBP, DBP, ABP, MAP and heart rate in fructose-fed rats receiving quercetin treatment (43). The mechanisms underlying the cardioprotective effects of quercetin against fructose-fed IR-induced abnormality, reduction in cardiac functions could be attributed to the ability of to improve insulin sensitivity, ability to help to maintain plasma insulin and glucose levels near normal also help to protect the heart against hyperglycemia-induced oxidative stress.

\section{CONCLUSION}

A conclusive finding of this study summarized, quercetin has insulin-sensitizing effects as well as improve metabolism in fructose-fed rats. It has additional better control of uric acid level and antioxidant potential with compare of standard treatment of metformin drug for IR state and its associated complications. Which could be explained on the basis of its antioxidant effect might help to prevent and also decrease the degree of myocardial injury as well as improve function in IR state. In view of these, quercetin could potentially be used as a drug to improve cardio-protection. More investigations should be conducted to elucidate the mechanisms at the molecular level and to test the effects of quercetin on vascular complications that accompany IR and metabolic syndrome.

\section{Acknowledgements}

The authors would like to thanks to the management committee of ASBASJS Memorial College of Pharmacy BELA (Ropar) and IK Gujral Punjab Technical University, Jalandhar, Kapurthala, Punjab, India, for providing facilities and registration to conduct the project work.

\section{Competing of interest statement}

We have declared that we have no competing or conflict of interest exists.

\section{REFERENCES}

1. Dornas W.C., de Lima W.G., Pedrosa M.L., Silva M.E.: Adv. Nutr. 13, 729 (2015).

2. Malik V.S., Hu F.B.: J. Am. Coll. Cardiol. 66, 1615 (2015).

3. Bantle J.P.: J. Nutr.139, 1263S (2009).

4. Meshkani R., Adeli K.: Clin. Biochem. 42, 1331 (2009).

5. Axelsen L.N., Lademann J.B., Petersen J.S., Holstein-Rathlou N.H., Ploug T. et al.: Am. J. Physiol. Regul. Integr. Comp. Physiol. 298, R1560 (2010).

6. Smit J.W., Romijn J.A.: Semin. Cardiothorac. Vasc. Anesth. 10, 215 (2006).

7. Mellor K.M., Bell J.R., Young M.J., Ritchie R.H., Delbridge L.M.: J. Mol. Cell. Cardiol. 50, 1035 (2011).

8. Zhang B.B., Zhou G., Li C.: Cell Metab. 9, 407 (2009).

9. Hardie D.G.: Am. J. Clin. Nutr. 93, 891S (2011).

10. Srivastava R.K., Pinkosky S.L., Filippov S., Hanselman J.C., Cramer C.T. et al.: J. Lipid Res. 53, 2490 (2012). 
11. Fogarty S., Hardie D.G.: Biochim. Biophys. Acta. 1804, 581 (2010).

12. Lee Y.S., Kim W.S., Kim K.H., Yoon M.J., Cho H.J. et al.: Diabetes 55, 2256 (2006).

13. Jeong S.M., Kang M.J., Choi H.N., Kim J.H., Kim J.I.: Nutr. Res. Pract. 6, 201 (2012).

14. Li Y., Yao J., Han C., Yang J., Chaudhry M.T. et al.: Nutrients 15, 167 (2016).

15. Hayek T., Fuhrman B., Vaya J., Rosenblat M., Belinky P. et al.: Arterioscler. Thromb. Vasc. Biol. 17, 2744 (1997).

16. Guo X.D., Zhang D.Y., Parry J., Liu K., Liu B.L.: Mol. Nutr. Food. Res. 57, 1037 (2013).

17. Eid H.M., Nachar A., Thong F., Sweeney G., Haddad P.S.: Pharmacogn. Mag. 41, 74 (2015).

18. Ahn J., Lee H., Kim S., Park J., Ha T.: Biochem. Biophys. Res. Commun. 373, 545 (2008).

19. Li M., Jiang Y., Jing W., Sun B., Miao C. et al.: Can. J. Physiol. Pharmacol. 91, 951 (2013).

20. Kravchuk E., Grineva E., Bairamov A., Galagudza M., Vlasov T.: Exp. Diabetes Res. 10, 1 (2011).

21. Shimoda I., Koizumi M., Shimosegawa T., ShishidoT., Ono T. et al.: Pancreas 8, 196 (1993).

22. Matthews D.R., Hosker J.P., Rudenski A.S., Naylor B.A., Treacher D.F. et al.: Diabetologia 28, 412 (1985).

23. Ohkawa H., Ohishi N., Yagi K.: Anal. Biochem. 95, 351 (1979).

24. Misra H.P., Fridovich I.: 1972. J. Biol. Chem. 247, 3170 (1972)

25. Maehly A.C., Chance B.: Biochem. Anal. 1, 357 (1954).

26. Rotruck J.T., Pope A.L., Ganther H.E., Swanson A.B., Hafeman D.G. et al.: Science 179, 588 (1973).
27. Hwang I.S., Ho H., Hoffman B.B., Reaven G.M.: Hypertension 10, 512 (1987).

28. Perez-Vizcaino F., Duarte J.: Mol. Aspects. Med. 31, 478 (2010).

29. Stark A.H., Timar B., Madar Z.: Eur. J. Nutr. 39, 229 (2000).

30. Fan S., Zhang Y., Sun Q., Yu L., Li M., Zheng B. et al.: J. Nutr. Biochem. 25, 702 (2014).

31. Lozano I., Werf1 R.V., Bietiger W., Seyfritz E., Peronet C. et al.: Nutr. Metab. 13, 2 (2016).

32. Haeri M.R., Izaddoost M., Ardekani M.R., Nobar M.R., White K.N.: Phytother. Res. 23, 61 (2009).

33. Soltani Z., Rasheed K., Kapusta D.R., Reisin E.: Curr. Hypertens. Rep. 15, 175 (2013).

34. Furukawa S., Fujita, Shimabukuro T.M., Waki M.I., Yamada Y. et al.: J. Clin. Invest. 114, 1752 (2004).

35. Luangaram S., Kukongviriyapan U., Pakdeechote P., Kukongviriyapan V., Pannangpetch P.: Food Chem. Toxicol. 45, 448 (2007).

36. Mellor K.M., Bell J.R., Ritchie R.H., Delbridge L.M.D.: P. Aust. Biochem. Soc. 43, 117 (2012).

37. Duan J., Wei G., Guo C., Cui J., Yan J. et al.: Am. J. Chin. Med. 43, 1159 (2015).

38. Mikolajczyk T.P., Nosalski R., Szczepaniak P., Budzyn K., Osmenda G. et al.: FASEB J. 30, 1987 (2016)

39. Kaur H., Parikh V., Sharma A., Singh M.: Pharmacol. Res. 36, 95 (1997).

40. Saponaro F., Sonaglioni A., Rossi A., Montefusco L., Lombardo M. et al.: Diabetes Res. Clin. Pract. 118, 21 (2016).

41. Sinha R., Srivastava S., Joshi A., Joshi U.J., Govil G.: Eur. J. Med. Chem. 22, 102 (2014).

42. Sak K.: Mini. Rev. Med. Chem. 14, 494 (2014).

43. Duarte J., Pérez-Palencia R., Vargas F., Ocete M.A., Pérez-Vizcaino F. et al.: Br. J. Pharmacol. 133, 117 (2001).

Received: 28.09 .2017 